\title{
Die Felchen des Alpnachersees
}

\author{
Von Per-Ole Svarvar und Rudolf Müller \\ Seenforschungslaboratorium der EAWAG/ETH, CH-6047 Kastanienbaum, Schweiz
}

Manuskript eingegangen am 4. März 1982

\begin{abstract}
The whitefish (Coregonus sp.) of Lake Alpnach (Switzerland)

In eutrophic Lake Alpnach, several forms of whitefish (Coregonus spp.) are found which show different growth rates. In recent years, immature whitefish of the fastgrowing form (local name: 'Felchen', here called 'Grossfelchen') have been caught in the nets used for fishing the slowgrowing form (local name: 'Albeli', here called 'Kleinfelchen'). The aim of this study was to identify the different forms of coregonids and to determine their growth rate, age of first maturation, and relative frequency in the catch with gill nets of different mesh sizes. While the separation of the different forms on the basis of meristic characters proved unsatisfactory, the growth during the first year of life was found best suited for this purpose. For the whitefish fishery in Lake Alpnach, the fastgrowing form is regarded as the most important one. Due to eutrophication, the whitefish now show a considerably faster growth than about 40 years ago. In addition, they mature at a younger age than before. The consequences of these findings for the management of the whitefish stocks in Lake Alpnach are discussed.
\end{abstract}

\section{INHALTSVERZEICHNIS}

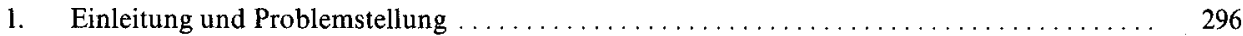



3. Material und Methoden ........................................... 297

3.1 Probenahmen ................................................. 297

3.2 Untersuchungsmethodik ............................................. 297

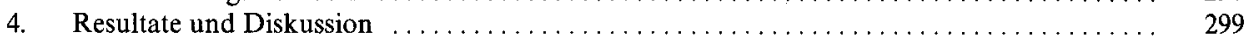

4.1 Trennung der verschiedenen Felchenformen . . . . . . . . . . . . . . . . . . . . . . . . . . 299

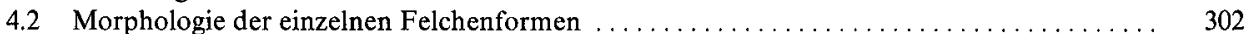

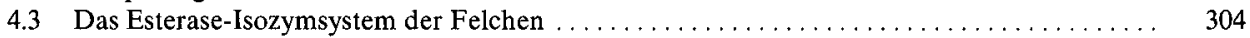



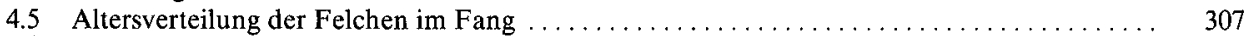

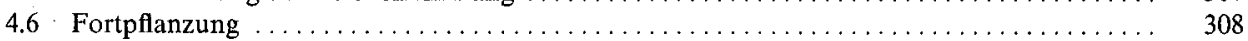

5. Konsequenzen für die Bewirtschaftung der Felchen im Alpnachersee . . . . . . . . . . . . 310

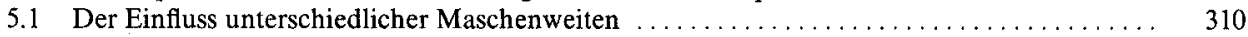

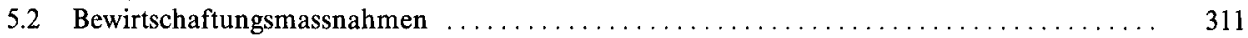

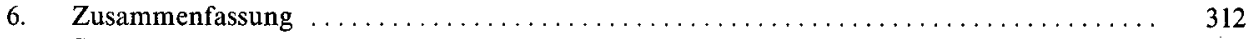

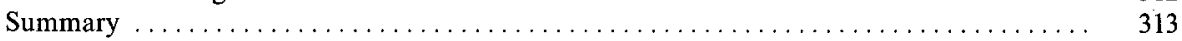

Résumé .................................................. 313

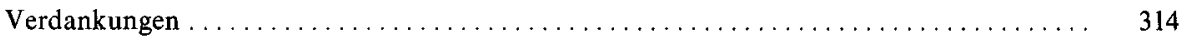

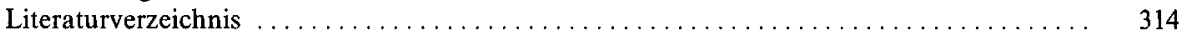




\section{Einleitung und Problemstellung}

Die wirtschaftlich wichtigsten Fische des Alpnachersees sind, wie in den meisten Schweizer Seen, die Felchen oder Coregonen. Nach Birrer und Schweizer [4] kommen folgende Coregonenformen vor:

- Albeli (Coregonus exiquus albellus, Fatio), auch Weissfisch genannt, und

- Balchen (Coregonus Schinzii helveticus var. lucernensis, Fatio), der aber praktisch nur zur Laichzeit gefangen wird.

Seit Ende der vierziger Jahre sind Blaufelchen (Coregonus wartmanni) aus dem Bodensee im Alpnachersee eingesetzt worden, nebst Felchen aus dem Sarner- und dem Sempachersee (Fischereiaufseher E. Odermatt und J. Muggli, persönliche Mitteilungen). Die Grossfelchen (in dieser Arbeit Bezeichnung für Balchen, Blaufelchen, Sarnerseefelchen und Sempacherseefelchen) sind schnellwüchsiger als die langsamwüchsigen Kleinfelchen (in dieser Arbeit Bezeichnung für Albeli) und haben deshalb seit Anfang der fünfziger Jahre grössere wirtschaftliche Bedeutung erlangt. Alle diese Felchenformen laichen im Alpnachersee, die Albeli auf dem Seegrund, die Grossfelchen meist in der Freiwasserzone, aber auch auf dem Seegrund.

Weil der Alpnachersee ein relativ seichter See ist, sind die Lebensräume der verschiedenen Felchenformen nicht deutlich voneinander getrennt. Deshalb werden in den sogenannten Albelinetzen unterschiedliche Mengen junger Grossfelchen gefangen, die noch nie abgelaicht haben, aber gleich gross sind wie die älteren Kleinfelchen. Die Mindestmaschenweite der Albelinetze von $28 \mathrm{~mm}$ wurde seit vielen Jahren beibehalten, obwohl die Wachstumsrate der Felchen u. a. wegen der Eutrophierung offensichtlich zugenommen hatte.

Das Ziel der vorliegenden Untersuchung war es daher, erst einmal die verschiedenen Felchenformen des Alpnachersees zu identifizieren und ihre Charakteristika wie Wachstum, Eintritt der Geschlechtsreife und relative Häufigkeit im Fang mit Netzen unterschiedlicher Maschenweite zu erfassen. Daraus sollten dann Empfehlungen für die optimale Nutzung der verschiedenen Felchenpopulationen abgeleitet werden. Um zu sehen, ob die gleichen Probleme auch im Vierwaldstättersee bestehen, wurde zusätzlich eine Anzahl Felchen aus dem Gebiet vor Stansstad und Hergiswil untersucht. In dieser Arbeit wird das Untersuchungsgebiet ohne Alpnachersee als Vierwaldstättersee bezeichnet. Im Vierwaldstättersee ist das Albeli (Kleinfelchen) von grosser wirtschaftlicher Bedeutung, die Grossfelchen dagegen sind weniger bedeutend.

\section{Charakterisierung des Alpnachersees}

Der Alpnachersee (Fläche $4,76 \mathrm{~km}^{2}$ ) ist mit $32 \mathrm{~m}$ maximaler Tiefe ein verhältnismässig seichter Voralpensee, der mit dem übrigen tieferen Vierwaldstättersee kanalartig verbunden ist. Den grössten Teil des Zuflusses erhält der Alpnachersee durch die Sarner Aa, die in den südlichen Teil des Sees mündet und ein Delta bildet. Nach Krummenacher [10] ist der Alpnachersee eutroph, während der Vierwaldstättersee als mesotroph gilt. Eine Zusammenstellung der hydrologischen 
und morphometrischen Daten des Alpnachersees findet sich bei Krummenacher [10] und Baccini [1].

Neben den Felchen ist auch der Hecht von wirtschaftlicher Bedeutung im Alpnachersee. Daneben werden Barsche, Trüschen, Seeforellen, Äschen, Zander und verschiedene Cypriniden gefangen.

\section{Material und Methoden}

\subsection{Probenahmen}

Vom November 1977 bis Dezember 1978 wurden in etwa monatlichen Intervallen total 701 Coregonen untersucht. Der grösste Teil entstammte dem Fang der Berufsfischer mit Kiemennetzen (Grund- und Schwebnetze von 28, 30, 38 und $50 \mathrm{~mm}$ Maschenweite). Ein kleinerer Teil der untersuchten Fische wurde mit eigenen Kiemennetzen mit Maschenweiten von 8, 10, 12, 14, 20 und $24 \mathrm{~mm}$ gefangen.

Zusätzlich hierzu wurde bei einigen Felchen der Wachstumsbeginn im Frühjahr 1979 bestimmt. Die Netze wurden jeweils am Nachmittag gesetzt und frühmorgens eingeholt. Folgende Tiefen wurden befischt:

\begin{tabular}{lll}
\hline Setztiefe $(\mathrm{m})$ & & \\
See & Grundnetze & Schwebnetze \\
\hline Alpnachersee & $15-30$ & $4-22$ \\
Vierwaldstättersee & $30-60$ & $2-13$ \\
\hline
\end{tabular}

\subsection{Untersuchungsmethodik}

\subsection{Biometrie}

Bei rund der ersten Hälfte (334 Fische) der untersuchten Felchen wurden folgende Kriterien verwendet: Totallänge, Körperlänge, Gewicht, Geschlecht und Reifegrad. Ausserdem wurden Schuppen sowie der erste rechte Kiemenbogen entnommen. Als Ort der Schuppenentnahme wählte man die Schuppenreihe unmittelbar unterhalb der Seitenlinie, auf der Höhe des vorderen Randes der Bauchflosse [16]. Bei der zweiten Hälfte der untersuchten Felchen (367 Fische) wurden zusätzlich folgende Merkmale berücksichtigt: Augendurchmesser, Kopflänge, Länge der Brustflosse, Länge der Rückenflossenbasis, Länge der Afterflossenbasis, Schwanzstielhöhe, Anzahl der Seitenlinienschuppen.

\subsection{Altersbestimmung}

Für die Identifikation eines Annulus (Jahresrings) stützten wir uns auf die von Tesch [20] definierten Kriterien. Mit Hilfe eines Projektionsmikroskops wurden die Abstände der einzelnen Annuli vom Zentrum aus in oraler Richtung gemessen. Das Alter der Fische mit abgeschlossenem Jahreswachstum wurde mit 1,2,3, .. angegeben, während man das Alter der Fische mit unvollendetem Jahreszuwachs mit $1+$, $2+, 3+, \ldots$ bezeichnete. 
An den Schuppen wurden zusätzlich die Skleriten (Zuwachsringe) zwischen den Annuli gezählt. Die Zahl der Skleriten ist ein geeignetes Merkmal für die Bestimmung der Zuwachsrate (Bilton [2], Pearson [14]).

\subsection{Berechnung des Längenwachstums}

Die Rückberechnung der Länge der Fische bei verschiedenem Alter erfordert die Kenntnis der formentypischen Beziehung zwischen Schuppenradius 1 und Fischlänge L.

Eine Grundvoraussetzung für eine korrekte Rückberechnung der Länge ist die Trennung der verschiedenen Felchenformen. Mit Hilfe der von Lea [12] entwickelten einfachen Rückberechnungsmethode können aber die Fischlängen ohne vorhergehende Trennung der verschiedenen Formen berechnet werden. Bei dieser Methode wird angenommen, dass der Schuppenradius und der Fisch im gleichen Verhältnis zueinander (allometrisch) wachsen. Mit Hilfe dieser Methode wurden die Fischlängen des ganzen Materials beim ersten vollendeten Jahr berechnet, um einen eventuellen arttypischen Unterschied im Wachstum zu erfassen. Dabei wurden Fische verschiedener Jahrgänge getrennt behandelt und ihre Längenklassenverteilung in Histogrammen dargestellt.

Nach der Trennung der Felchenformen konnte die tatsächliche Beziehung zwischen Schuppenradius und Fischlänge der verschiedenen Formen mit Hilfe von Eichkurven dargestellt werden. Die Wachstumsbeschreibungen erfolgten nach der von Rufli [16] beschriebenen Methode.

Die mittleren Jahresendlängen wurden für alle Felchen, getrennt nach Formenzugehörigkeit, Geschlecht, Altersklasse und Jahrgang, berechnet. Daneben untersuchte man den mittleren jahreszeitlichen Zuwachs bei einem Teil der Fische.

\subsection{Elektrophoretische Untersuchungen}

Die Untersuchungen der Proteincharakteristika bei Fischen mit Hilfe von elektrophoretischen Verfahren bietet unter Umständen eine wertvolle Ergänzung bei systematischen und populationsgenetischen Analysen. An Leberproben von 112 Felchen aus beiden Seeteilen wurde deshalb ein Esterase-Isozymsystem untersucht. M. Himberg und B. Nyholm an der Biologischen Abteilung der Abo Akademi in Finnland führten die Untersuchung durch. Ihre Methodik ist eine Modifikation der von Ferguson [7] und Skog [17] verwendeten Technik und soll daher hier nur kurz beschrieben werden.

Die Lebern wurden laichreifen Fischen entnommen, homogenisiert und über Nacht bei $-15^{\circ} \mathrm{C}$ eingefroren. Tags darauf wurden sie wieder aufgetaut und mit dem gleichen Volumen Tris-Zitronensäurepuffer $(\mathrm{pH} 7,5)$, mit destilliertem Wasser im Verhältnis von 1:8 verdünnt, versetzt. Mit Hilfe von Filterpapierstückchen brachte man die Leberproben auf die Gelplatte. Für die Elektrophorese verwendete man ein diskontinuierliches Puffersystem. Tris-Zitronensäure ( $\mathrm{pH} \mathrm{7,5)} \mathrm{diente} \mathrm{als} \mathrm{Gelpuf-}$ fer und Natriumhydroxid-Borsäure $(\mathrm{pH} \mathrm{8,7)}$ als Elektrolytpuffer. Die Elektrophorese dauerte etwa 3,5 Stunden bei einer Temperatur zwischen 2 und $4{ }^{\circ} \mathrm{C}$. 
Die Esterasen wurden mit Hilfe einer Färbelösung (15 mg $a$-Naphtyl-Propionat in $1 \mathrm{ml}$ Wasser-Azeton-Lösung und $30 \mathrm{mg}$ Fast-Garnet-GBC-Salz in $50 \mathrm{ml} 0,1 \mathrm{M}$ Phosphatpuffer, $\mathrm{pH}$ 6,5) sichtbar gemacht. Die Fixierung erfolgte in einer Lösung mit 9 Teilen 70prozentigem Alkohol und 11 Teilen 20prozentiger Essigsäure.

\section{Resultate und Diskussion}

\subsection{Trennung der verschiedenen Felchenformen}

\subsection{Anzahl Kiemenreusendornen}

Nach Svaerdson [19] wird die Zahl der Kiemenreusendornen sehr wenig von der Umwelt beeinflusst und ist daher ein ideales Merkmal zur Untersuchung der Variabilität bei Felchen. Rufli [15] hat dagegen gefunden, dass sich die Reusendornenzahl bei einigen Felchenformen im Vergleich zu früheren Daten um etwa vier Einheiten erhöht hat.

Aufgrund der Verteilung der Kiemenreusendornen bei den Alpnacherseefelchen könnte man erwarten, dass bei jenen Fischen, die in den Jahren 1974, 1975 und 1976 geschlüpft sind, zwei verschiedene Formen vorkommen: eine Form mit einer mittleren Kiemenreusendornenzahl von etwa 33 und eine solche mit rund 41. Die Felchen des Jahrgangs 1977 zeigen eine Verteilung mit nur einem Maximum bei etwa 37 Kiemenreusendornen. Die Felchen des Vierwaldstättersees besitzen beinahe alle hohe Kiemenreusendornenzahlen mit einem Mittelwert um 40.

Da sich die Verteilungen der Kiemenreusendornenzahlen stark überlappen, können die Felchen nicht aufgrund dieses Merkmals allein getrennt werden.

\subsection{Längenwachstum und Anzahl Skleriten im ersten Jahr}

Nach Karbe [9] lassen sich die Bodenseefelchen einigermassen durch die Kombination der beiden Merkmale Reusendornenzahl und Wachstum trennen. Nach Hartmann und Knoepfler [8] dagegen ist das Längenwachstum der Fische «extrem milieuabhängig und deshalb als taxonomisches Kriterium denkbar ungeeignet», was vor allem für den Vergleich bestimmter Formen aus verschiedenen Seen gelten dürfte.

Abb. 1 zeigt die Wachstumsverteilung der Alpnacherseefelchen nach dem ersten Lebensjahr, nach Jahrgängen getrennt. Die Jahrgänge 1974, 1975 und 1976 lassen sich deutlich in eine schnellwüchsige und eine langsamwüchsige Form trennen. Der Jahrgang 1977 liegt - wie bei den Kiemenreusendornen - auch im Wachstum zwischen den beiden obigen Extremen. Der grösste Teil der Vierwaldstätterseefelchen scheint aufgrund der Wachstumsverteilung im ersten Jahr dem langsamwüchsigen Typus anzugehören. Als Grenze zwischen langsam- und schnellwüchsigen Felchen wählte man den Wert von $120 \mathrm{~mm}$ Länge (unkorrigiert, nach Lea [12]) nach einem Lebensjahr. Die mittleren Kiemenreusendornenzahlen wurden nun für die beiden Wachstumsformen des Alpnachersees, nach Jahrgängen getrennt, berechnet und mit Hilfe des t-Tests miteinander verglichen (Tab. 1). Die Wahl eines Grenz- 

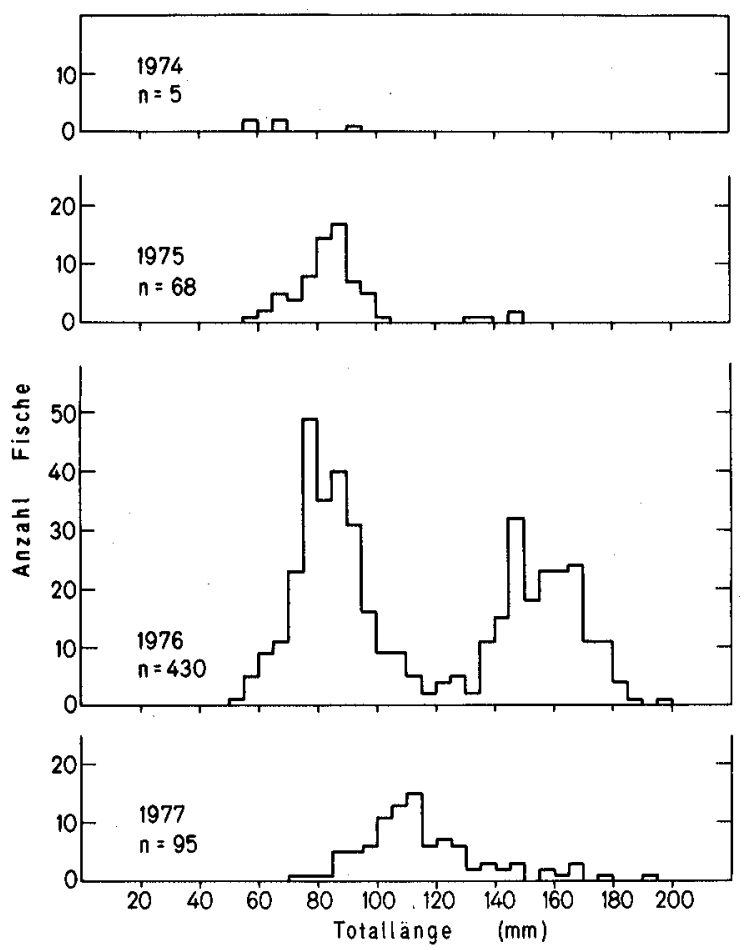

Abb. 1. Längenverteilung der Alpnacherseefelchen nach dem ersten Lebensjahr.

Figure 1. Length frequency distribution of Coregonus spp. of Lake Alpnach after the first year.

Tabelle 1. Mittlere Kiemenreusendornenzahlen mit Standardabweichung für die beiden Wachstumstypen des Alpnachersees, nach Jahrgängen getrennt. $\mathrm{KM}=$ mittlere

Kiemenreusendornenzahl, ST = Standardabweichung, $\mathrm{P}=$ Wahrscheinlichkeit für «nicht getrennt». Table 1. Mean number of gillrakers with standard deviation for both growth types of Lake Alpnach, separated according to year classes.

\begin{tabular}{lllllllll}
\hline Länge im & Jahrgang & & & & & & & \\
ersten & 1974 & & 1975 & & 1976 & & 1977 & \\
Jahr (mm) & KM & ST & KM & ST & KM & ST & KM & ST \\
\hline$<120$ & 41,2 & 1,48 & 40,9 & 3,29 & 40,2 & 2,42 & 37,3 & 3,43 \\
$>120$ & - & - & 31,5 & 2,38 & 33,0 & 3,07 & 35,3 & 4,61 \\
\hline p & & & $<0,001$ & & $<0,001$ & & $<0,005$ & \\
\hline
\end{tabular}

wertes von $135 \mathrm{~mm}$ Länge im ersten Jahr änderte nichts an den Mittelwerten der Kiemenreusendornenzahlen.

Pearson [14] und Bilton [2] haben gezeigt, dass das Verhältnis zwischen Wachstum und Skleritenzahl proportional ist. Die Häufigkeitsverteilung der Skleriten entspricht denn auch im wesentlichen der Wachstumsverteilung sowohl im Alpnachersee als auch im Vierwaldstättersee. Die schnellwüchsigen Felchen haben durchwegs 
eine höhere Anzahl Skleriten als die langsamwüchsigen Felchen, was zeigt, dass das Verhältnis zwischen Skleritenzahl und Wachstum fast linear verläuft.

Die Wachstumsgeschwindigkeit trennt also die Felchen des Alpnacher- und Vierwaldstättersees in zwei Formen, welche in dieser Arbeit fortan als Klein- und Grossfelchen bezeichnet werden (siehe Abb.2). Auch Steinmann [18] hat diese Bezeichnung für die Vierwaldstätterseefelchen aufgrund der Fanggrösse benützt.

Eine kleine Anzahl grosser und älterer Felchen (Totallänge $>320 \mathrm{~mm}$ ), die während des ersten Jahres schlecht gewachsen waren $(<120 \mathrm{~mm})$, wurden ebenfalls als Grossfelchen betrachtet. Diese Erscheinung, dass einzelne Fische anfangs schlecht wachsen und damit im normalen Fangalter nicht weggefangen werden, wird auch als Lees Phänomen bezeichnet (Tesch [20]).

Weil die 95 Alpnacherseefelchen des Jahrgangs 1977 schwer zu klassifizieren waren, wurden sie bei der weiteren Behandlung des Felchenmaterials weggelassen. Ein Teil davon könnten Hybriden oder Zwischenformen sein. Svaerdson [19] hatte gefunden, dass die Nachkommen zweier Individuen mit hoher und niedriger Kiemenreusendornenzahl eine mittlere Anzahl aufweisen.

Die zahlenmässige Verteilung der verschiedenen gefangenen Felchenformen sieht nach der Trennung wie folgt aus (ohne Jahrgang 1977):

$\begin{array}{lll}\text { Form: } & \text { Alpnachersee: } & \text { Vierwaldstättersee: } \\ \text { Kleinfelchen } & 302 & 97 \\ \text { Grossfelchen } & \frac{201}{503} & \frac{6}{103}\end{array}$

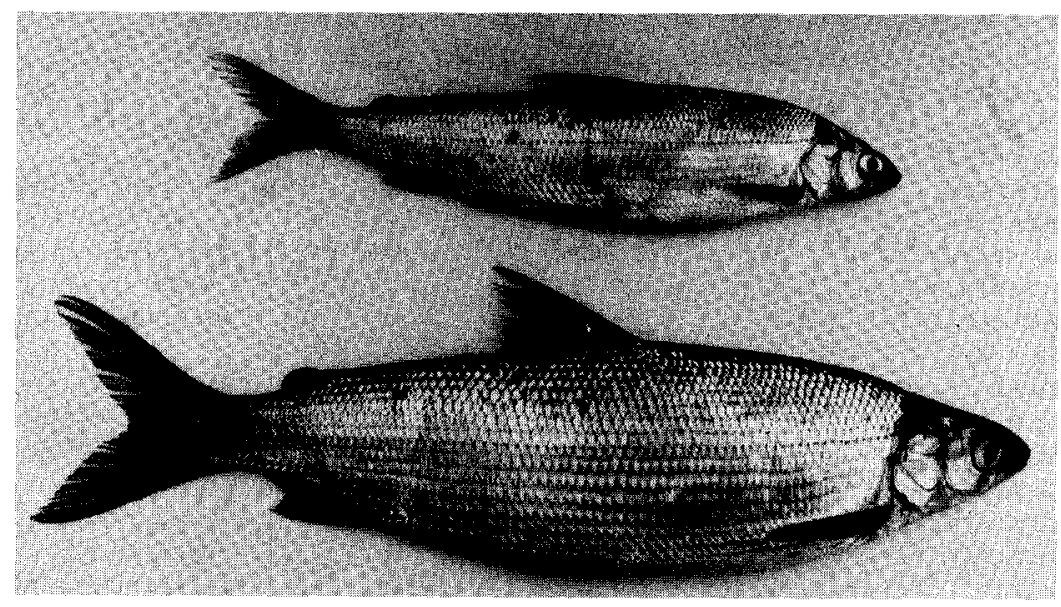

Abb.2. Felchen aus dem Alpnachersee. Oben Kleinfelchen, Totallänge $244 \mathrm{~mm}$, Gewicht $113 \mathrm{~g}$, Anzahl Kiemenreusendornen 41, Alter 2+. Unten Grossfelchen, Totallänge $345 \mathrm{~mm}$, Gewicht $370 \mathrm{~g}$, Anzahl Kiemenreusendornen 33, Alter 2+.

Figure 2. Coregonus spp. of Lake Alpnach. Above 'Kleinfelchen', total length $244 \mathrm{~mm}$, weight $113 \mathrm{~g}$, number of gillrakers 41, age $2+$. Below 'Grossfelchen', total length $345 \mathrm{~mm}$, weight $370 \mathrm{~g}$, number of gillrakers 33 , age $2+$. 


\subsection{Morphologie der einzelnen Felchenformen}

\subsection{Zahl der Kiemenreusendornen}

In Tabelle 2 sind die Verteilungen der Kiemenreusendornenzahl für alle Felchenformen und beide Seen, in Abb. 3 jene der Alpnacherseefelchen dargestellt. Innerhalb je eines Seeteils sind demnach die Gross- und Kleinfelchenpopulationen getrennt $(p<0,001)$, nicht aber die Gross- bzw. Kleinfelchenpopulationen der beiden Seeteile.

Die Zahl der Kiemenreusendornen bei den Kleinfelchen liegt um etwa zwei Einheiten höher als die in der Literatur angegebenen Werte für Albeli. Rufli [15] vermutet als mögliche Ursachen hiefür die natürliche Selektion, veränderte Umweltbedingungen oder die höhere Wachstumsrate (siehe unten).

Daneben könnten als Folge des beschleunigten Wachstums heute auch die kleinen Dornen mitgezählt werden, die bei dem früheren, langsamwüchsigen Albeli nicht sichtbar waren. Man ist daher zur Annahme berechtigt, dass das Albeli (der Weissfisch) und der Kleinfelchen aufgrund der Kiemenreusendornenzahlen die gleiche Form sind. Aus dem gleichen Grund darf man annehmen, dass die Grossfelchen den Balchen und die von auswärts eingeführten Felchenformen umfassen.

Tabelle 2. Verteilung der Kiemenreusendornenzahl bei den verschiedenen einheimischen und eingeführten Felchenformen des Alpnacher- und Vierwaldstättersees.

Table 2. Distribution of gillrakers of different native and introduced forms of Coregonus spp.

\begin{tabular}{|c|c|c|c|c|}
\hline & \multicolumn{3}{|c|}{ Anzahl Kiemenreusendornen } & \multirow[t]{2}{*}{ Quelle } \\
\hline & Min. & Mittel & Max. & \\
\hline \multicolumn{5}{|l|}{ Kleinfelchen des } \\
\hline Alpnachersees & 29 & 40,5 & 47 & Vorliegende Arbeit \\
\hline \multicolumn{5}{|l|}{ Albeli, Weissfisch } \\
\hline \multicolumn{5}{|l|}{ Kleinfelchen des } \\
\hline Vierwaldstättersees & 36 & 40,8 & 45 & Vorliegende Arbeit \\
\hline \multicolumn{5}{|l|}{ Albeli, Weissfisch des } \\
\hline Vierwaldstättersees & 35 & 38,7 & 45 & Birrer und Schweizer [3] \\
\hline \multicolumn{5}{|l|}{ Grossfelchen des } \\
\hline Alpnachersees & 24 & 33,2 & 45 & Vorliegende Arbeit \\
\hline \multicolumn{5}{|l|}{ Grossfelchen des } \\
\hline Vierwaldstättersees & 27 & 34,3 & 39 & Vorliegende Arbeit \\
\hline \multicolumn{5}{|l|}{ «Winterlaichende } \\
\hline \multicolumn{5}{|l|}{ Grossfelchen» des } \\
\hline Alpnachersees* & 29 & & 38 & Steinmann [18] \\
\hline Edelfisch & 32 & 35,5 & 41 & Birrer und Schweizer [5] \\
\hline Balchen & 20 & 25,5 & 31 & Birrer und Schweizer [6] \\
\hline \multicolumn{5}{|l|}{ Blaufelchen aus } \\
\hline dem Bodensee & 29 & 36,2 & 46 & Steinmann [18] \\
\hline Sarnerseefelchen & & 36,0 & & Steinmann [18] \\
\hline Sempacherseefelchen & 26 & 33,5 & 38 & Steinmann [18] \\
\hline
\end{tabular}

* Wahrscheinlich Blaufelchen aus dem Bodensee. 


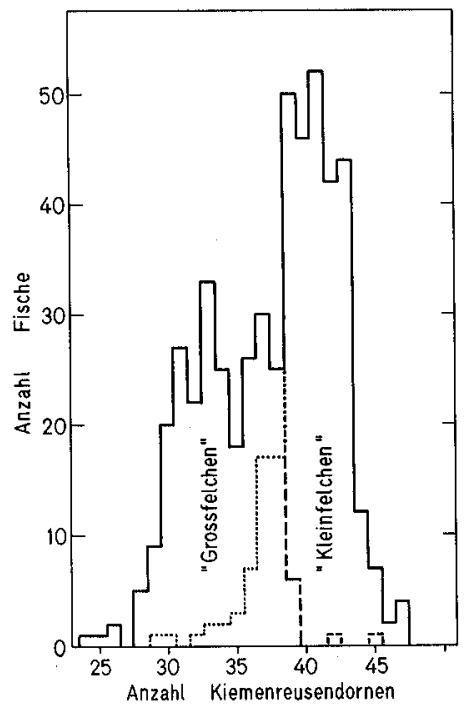

Abb.3. Häufigkeitsverteilung der Anzahl Kiemenreusendornen bei den Alpnacherseefelchen.

Figure 3. Frequency distribution of gillrakers of coregonids of Lake Alpnach.

\subsection{Andere morphologische Merkmale}

Die Resultate der Untersuchung weiterer morphologischer Merkmale sind in den Tabellen 3 und 4 zusammengestellt.

Tabelle 3. Morphologische Merkmale der verschiedenen Felchenformen.

Table 3. Morphological characteristics of the different forms of Coregonus spp.

\begin{tabular}{|c|c|c|c|c|c|c|c|c|c|c|c|c|}
\hline \multirow[t]{2}{*}{ Merkmal } & \multicolumn{11}{|c|}{ Felchenform } & \\
\hline & M & ST & $\mathrm{N}$ & $\mathrm{M}$ & ST & $\mathrm{N}$ & $\mathbf{M}$ & ST & $\mathrm{N}$ & $\mathbf{M}$ & ST & $\mathrm{N}$ \\
\hline \multicolumn{13}{|l|}{ Augengrösse/ } \\
\hline Totallänge $(\times 100)$ & 3,88 & 0,28 & 165 & 3,40 & 0,41 & 12 & 3,84 & 0,23 & 97 & 3,41 & 0,13 & 6 \\
\hline Kopflänge/ & & & & & & & & & & & & \\
\hline Totallänge $(\times 100)$ & 17,1 & 0,88 & 165 & 16,2 & 0,72 & 12 & 16,9 & 0,58 & 97 & 15,8 & 0,42 & 6 \\
\hline \multicolumn{13}{|l|}{ Brustflossenlänge/ } \\
\hline Totallänge $(\times 100)$ & 13,6 & 0,86 & 165 & 12,6 & 0,81 & 12 & 13,5 & 0,61 & 97 & 12,3 & 0,34 & 6 \\
\hline \multicolumn{13}{|l|}{ Rückenflossenbasis/ } \\
\hline Totallänge $(\times 100)$ & 9,30 & 0,79 & 165 & 10,1 & 0,76 & 12 & 9,38 & 0,61 & 97 & 10,4 & 0,92 & 6 \\
\hline \multicolumn{13}{|l|}{ Afterflossenbasis/ } \\
\hline Totallänge $(\times 100)$ & 10,8 & 0,85 & 165 & 10,6 & 0,79 & 12 & 10,8 & 0,74 & 97 & 10,6 & 0,98 & 6 \\
\hline \multicolumn{13}{|l|}{ Schwanzsticlhöhe/ } \\
\hline Totallänge $(\times 100)$ & 6,04 & 0,38 & 165 & 6,51 & 0,36 & 12 & 5,92 & 0,33 & 97 & 6,65 & 0,53 & 6 \\
\hline \multicolumn{13}{|l|}{ Anzahl } \\
\hline Seitenlinienschuppen & 86,2 & 4,28 & 165 & 88,8 & 3,71 & 12 & 86,1 & 4,08 & 97 & 91,5 & 5,31 & 6 \\
\hline
\end{tabular}

Abkürzungen: $\mathrm{KA}=$ Kleinfelchen des Alpnachersees, $\mathrm{GA}=$ Grossfelchen des Alpnachersees, $\mathrm{KV}=$ Kleinfelchen des Vierwaldstättersees, $\mathrm{GV}=$ Grossfelchen des Vierwaldstättersees, $M=\mathrm{Mittel}$, ST = Standardabweichung, $\mathrm{N}=$ Anzahl. 
Tabelle 4. Vergleich morphologischer Merkmale zwischen den verschiedenen Felchenformen. Für Abkürzungen siehe Tabelle 3.

Table 4. Comparison of morphological characteristics between the different forms of Coregonus spp. For abbreviations see table 3 .

\begin{tabular}{lllll}
\hline Merkmal & Testpaar & & & \\
& KA/GA & KA/KV & GA/GV & KV/GV \\
\hline Relative Augengrösse & +++ & - & - & +++ \\
Relative Kopflänge & +++ & + & - & +++ \\
Relative Brustflossenlänge & +++ & - & - & +++ \\
Relative Rückenflossenbasis & ++ & - & - & +++ \\
Relative Afterflossenbasis & - & - & - & - \\
Relative Schwanzstielhöhe & +++ & + & - & +++ \\
Anzahl Seitenlinienschuppen & + & - & - & ++ \\
\hline
\end{tabular}

Bedeutung der Zeichen: - Kein statistisch gesicherter Unterschied; $+p<0,05$, Unterschied statistisch gesichert; $++p<0,01$, Unterschied statistisch gut gesichert; $+++p<0,001$, Unterschied statistisch sehr gut gesichert.

Es zeigt sich auch hier, dass Gross- und Kleinfelchen innerhalb eines Seeteils unterscheidbar sind, nicht aber einander entsprechende Formen in den beiden Seeteilen.

\subsection{Das Esterase-Isozymsystem der Felchen}

Die Esteraseaktivität der untersuchten Fischlebern war sehr gering. Deshalb konnten die Leberesterasezymogramme nur bei 44 Felchen aus beiden Seeteilen interpretiert werden, wobei die Alpnachersee- und Vierwaldstätterseefelchen hier zusammen behandelt werden. Eine mögliche Ursache der niedrigen Aktivität war die Laichreife bei fast allen Tieren (Himberg, persönliche Mitteilung).

Das Leberesterasezymogramm besteht aus vier verschiedenen Zonen (Abb.4): Die ersten zwei Zonen kommen bei den meisten untersuchten Felchen vor, aber sie werden nicht interpretiert, weil ihre Variation nicht mit einem bestimmten genetischen Modell gekoppelt werden konnte. Die dritte Zone ist meistens unsichtbar,

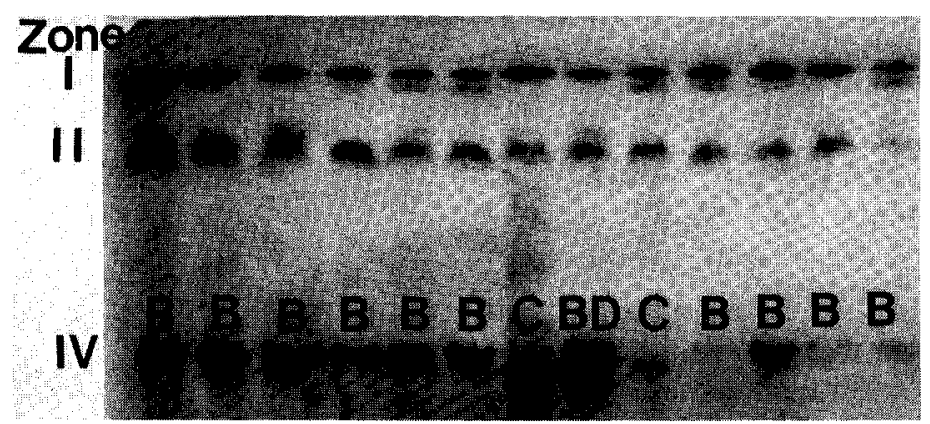

Abb. 4. Leberesterasezymogramme. Die Buchstaben bezeichnen die verschiedenen Phänotypen. Figure 4. Esterase zymograms of liver homogenates. The letters characterize the different phenotypes. 
weil die Enzyme darin sehr schnell abgebaut werden. Die vierte, langsamwandernde Zone ist am besten untersucht. Skog [17] hat in dieser Zone vier verschiedene Banden, entsprechend Allelen, festgestellt. Dieses System kann genetisch durch das Auftreten von vier kodominanten Allelen an einem Locus (in diesem Fall Zone IV) erklärt werden. Bei einem Individuum kommen eine (homozygot) oder zwei Banden (heterozygot) vor. Bei den untersuchten Felchen (Tab.5) wurden nur vier verschiedene Phänotypen angetroffen, während Skog [17] alle theoretisch möglichen Kombinationen gefunden hat.

BB und CC bezeichnen die Phänotypen B bzw. C (Homozygoten) bei der Berechnung der Allelfrequenz. Unterschiede in der Allelfrequenz zwischen den beiden Formen wurden mit dem Chi-Quadrat-Test geprüft, wobei ein statistisch gesicherter Unterschied $(\mathrm{p}<0,001)$ festgestellt wurde.

\subsection{Das Längenwachstum}

4.41 Beziehung zwischen dem Schuppenradius und der Totallänge der Fische

Die Konstanten für die Beziehung $1=\mathrm{a}+\mathrm{bL}+\mathrm{cL}^{2}$ zwischen dem Schuppenradius 1 und der Totallänge $L$ der Fische bei den verschiedenen Felchenformen sind folgende:

\begin{tabular}{lllr}
\hline Felchenform & Konstanten & \\
& $\mathrm{a}$ & $\mathrm{b}$ & \multicolumn{1}{c}{$\mathrm{c}$} \\
\hline Kleinfelchen des Alpnachersees & $-8,62$ & 0,34 & $2,7 \times 10^{-4}$ \\
Grossfelchen des Alpnachsees & $-9,66$ & 0,38 & $1,8 \times 10^{-4}$ \\
Kleinfelchen des Vierwaldstättersees & $-11,1$ & 0,45 & $-1,4 \times 10^{-4}$ \\
Grossfelchen des Vierwaldstättersees & $-8,43$ & 0,33 & $2,7 \times 10^{-4}$ \\
\hline
\end{tabular}

Nach Rufli [16] weichen die Beziehungen 1/L bei verschiedenen Felchenformen innerhalb desselben Sees bedeutend stärker voneinander $a b$ als jene der entsprechenden Formen aus beiden Seen, was sich hier nur zum Teil bestätigte.

\subsection{Das Längenwachstum der Alpnacher- und Vierwaldstätterseefelchen}

Die mittleren Jahresendlängen der Alpnacherseefelchen mit den entsprechenden 95\%-Vertrauensgrenzen sind in Abb.5 dargestellt. Wie auch Tabelle 6 zeigt, wachsen die Kleinfelchen heute viel schneller als die Albeli vor etwa 40 Jahren. Sie

Tabelle 5. Phänotypverteilung aufgrund elektrophoretischer Untersuchungen.

Table 5. Distribution of phenotypes based on electrophoretic examinations.

\begin{tabular}{lllll}
\hline Phänotypus & Kleinfelchen & \multicolumn{2}{c}{ Grossfelchen } \\
& Anzahl & $\%$ & Anzahl & $\%$ \\
\hline B & 33 & 97,1 & 5 & 50,0 \\
BC & - & - & 1 & 10,0 \\
C & 1 & 2,9 & 3 & 30,0 \\
BD & - & - & 1 & 10,0 \\
\hline
\end{tabular}


Tabelle 6. Mittlere Jahresendlängen der Alpnachersee- und Vierwaldstätterseefelchen. Abkürzungen siehe Tabelle 3.

Table 6. Mean annual length of the Coregonus spp. of Lake Alpnach and Lake Lucerne. For abbreviations see table 3 .

\begin{tabular}{lllllllllllll}
\hline Jahr & \multicolumn{1}{l}{ Mittlere Jahresendlängen (mm) } & \multicolumn{1}{c}{ GA } & \multicolumn{1}{c}{ KV } & & & GV & \\
& KA & \multicolumn{1}{l}{ M } & M & ST & N & M & ST & N & M & ST & N \\
\hline 1 & 119,4 & 12,4 & 302 & 186,0 & 19,7 & 201 & 123,9 & 13,0 & 97 & 157,2 & 29,1 & 6 \\
2 & 214,5 & 18,9 & 239 & 283,3 & 38,1 & 28 & 215,8 & 15,2 & 96 & 293,6 & 48,2 & 5 \\
3 & 257,8 & 11,1 & 11 & 336,9 & 61,1 & 3 & 255,3 & 9,3 & 10 & 357,7 & 20,8 & 2 \\
\hline
\end{tabular}

erreichen jetzt innerhalb von zwei Jahren durchschnittlich die gleiche Länge wie früher in vier Jahren. Als Hauptursache dieser wesentlich besseren Wachstumsrate ist die seither stark fortgeschrittene Eutrophierung des Alpnachersees anzunehmen. Zwischen den beiden Formen eines Sees gibt es somit bezüglich des Wachstums durchgehend statistisch gesicherte Unterschiede.

Der Kleinfelchen zeigt eine ähnliche Wachstumskurve wie der «Brienzlig» des Thunersees, während die Wachstumskurve der Grossfelchen ungefähr jener des «Albocks» aus diesem See entspricht (Rufli [16]). Im Vergleich zum Blaufelchen des Bodensees wächst der Grossfelchen des Alpnachersees bis zum ersten Jahr ungefähr gleich gut, nachher etwas langsamer.



Abb.5. Mittlere Jahresendlängen der Alpnacherseefelchen mit den entsprechenden 95\%-

Vertrauensgrenzen. Zum Vergleich die von Birrer und Schweizer [4] gemessenen Totallängen der Albeli.

Figure 5. Mean annual lengths of coregonids of Lake Alpnach, with 95\% confidence limits, compared with the total lengths of 'Albeli' given by Birrer and Schweizer [4]. 
Die Grossfelchen des Alpnachersees wachsen im ersten Jahr viel schneller als die Kleinfelchen, im zweiten und dritten Jahr ist der Unterschied gering. Die jährlichen Schwankungen des Zuwachses bei beiden Formen sind relativ gering.

Bei den Kleinfelchen des Alpnachsees wachsen die Weibchen während der ersten zwei Jahre etwas schneller als die Männchen $(p<0,001)$. Für das dritte Jahr gibt es keinen statistisch gesicherten Unterschied. Bei den Grossfelchen des Alpnachersees hingegen wachsen die Männchen im ersten Jahr schneller, während sich für das zweite und dritte Jahr keine signifikanten Unterschiede ergeben. Bei den Kleinbzw. Grossfelchen des Vierwaldstättersees wurden keine statistisch gesicherten Wachstumsunterschiede zwischen den Geschlechtern gefunden.

\subsection{Der jahreszeitliche Zuwachs}

Dieser wurde an 176 Kleinfelchen aller Altersklassen aus dem Alpnachersee im Jahre 1978 untersucht (Abb.6). Er wurde als Differenz zwischen der Länge bei der letzten Annulusbildung und der Länge beim Fang bestimmt. Von elf am 19. Mai 1978 gefangenen Felchen hatten neun bereits zu wachsen begonnen. Bei den elf am 25. April 1979 zusätzlich gefangenen Felchen dagegen gab es noch keine neuen Zuwachsringe. Bei den zwei am 17. Mai 1979 gefangenen Fischen hatte das Wachstum dagegen schon eingesetzt. Dies zeigt, dass die Felchen des Alpnachersees in der ersten Hälfte Mai zu wachsen beginnen. Der Unterschied im Zuwachs zwischen den im November und den im Dezember gefangenen Fischen (Abnahme) ist wahrscheinlich ein Artefakt, eine Folge der grössenselektiven Fangmethode also.

Rufli [16] hat bei den Thuner- und Bielerseefelchen vergleichsweise festgestellt, dass sich die Wachstumsperiode von Anfang Mai bis Oktober erstreckt.

\subsection{Altersverteilung der Felchen im Fang}

Die Häufigkeitsverteilung der Altersklassen bei den untersuchten Alpnacherseefelchen ist in Abb. 7 wiedergegeben (nur Maschenweite $\geqslant 28 \mathrm{~mm}$ ).

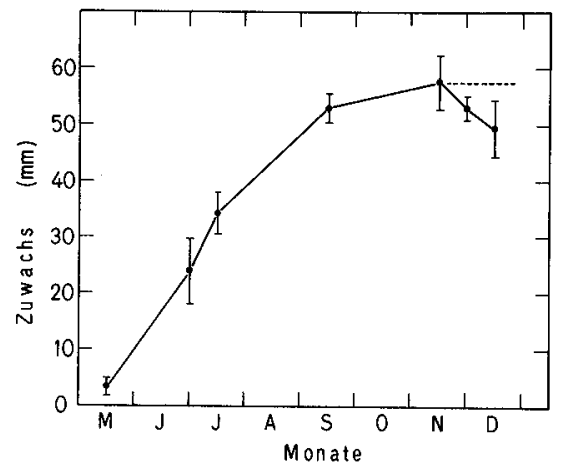

Abb. 6. Jahreszeitlicher Zuwachs bei den Kleinfelchen des Alpnachersees.

Figure 6. Seasonal growth of the 'Kleinfelchen' of Lake Alpnach. 


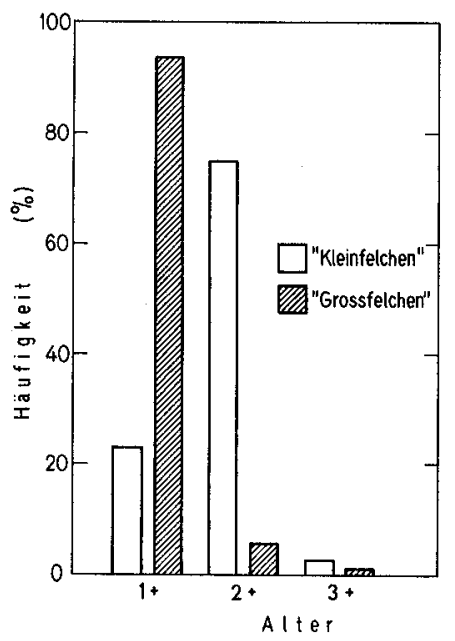

Abb.7. Altersverteilung der Alpnacherseefelchen: Fische aus Netzen mit Maschenweiten von $\geq 28 \mathrm{~mm}$. Figure 7. Age frequency distribution of coregonids of Lake Alpnach. Catch with mesh sizes of $\geq 28 \mathrm{~mm}$.

Danach werden die Kleinfelchen heute zum grössten Teil $(74,9 \%)$ im dritten Jahr bzw. als dreijährige, die Grossfelchen dagegen zu 93,5\% im zweiten Jahr bzw, als zweijährige gefangen, wobei die Versuchsfänge mehrheitlich Fische mit abgeschlossenem Jahreswachstum lieferten.

Die von Birrer und Schweizer [4] untersuchten Albeli zeigten demgegenüber eine ganz andere Altersverteilung als die heutigen Kleinfelchen: $70 \%$ des damaligen Fanges bestanden aus Fischen im fünften Lebensjahr!

Bei den Kleinfelchen sind die Verhältnisse im Vierwaldstättersee ähnlich wie im Alpnachersee. Der Umfang des Grossfelchenmaterials hingegen war zu klein, als dass damit hätten Vergleiche angestellt werden können.

\subsection{Fortpflanzung}

\subsection{Laichzeiten und Laichplätze}

Nach Beobachtungen der Berufsfischer beginnen die Kleinfelchen des Alpnachersees Anfang November zu laichen. Die Laichzeit dauert normalerweise bis Mitte Dezember. Die vorliegende Untersuchung hat ergeben, dass die Kleinfelchen, wie auch die Grossfelchen, von Ende November bis Anfang Januar laichen (Balchen Ende November bis Ende Dezember, Blaufelchen Anfang Dezember bis Anfang Januar). Dabei erreichen die Männchen der Alpnacherseefelchen die Laichreife offenbar vor den Weibchen. Ferner scheinen die Felchen des Vierwaldstättersees später zu laichen als jene im Alpnachersee, vermutlich wegen der langsameren Abkühlung des Wassers.

Nach unseren bisherigen Kenntnissen laichen die Kleinfelchen auf dem Seegrund, die Grossfelchen in höheren Wasserregionen: der Balchen in der Uferzone bis zur 
Halde, der Blaufelchen in der Freiwasserregion. Unsere Untersuchungen zeigten nun aber, dass der grösste Teil der Grossfelchen des Alpnachersees während der Laichzeit in Grundnetzen in Tiefen von 15 bis $25 \mathrm{~m}$ gefangen wurde. Überhaupt konnte - zumindest während der Laichzeit - kein grösserer Unterschied bezüglich des Lebensraums zwischen den beiden Felchenformen des Alpnachersees festgestellt werden.

$\mathrm{Ob}$ sich die Alpnacherseefelchen noch natürlich vermehren, ist nicht untersucht worden. Einige Gebiete des Seegrundes sollten noch genügend gute Bedingungen für die natürliche Fortpflanzung aufweisen. Diese Gebiete liegen vor dem Ausfluss des Alpnachersees (Kies-Sand-Anteil des Sediments bis 70\%) und zwischen den Mündungen der Sarner Aa und der Chli Schliere, mit einem Kies-Sand-Anteil des Sediments von bis zu 15\% (M. Sturm, persönliche Mitteilung).

\subsection{Geschlechtsverhältnisse}

Das Geschlechtsverhältnis (Männchen:Weibchen) bei den untersuchten Felchen zeigt folgendes Bild:

\begin{tabular}{lllll}
\hline & $\begin{array}{l}\text { Alpnachersee } \\
\text { Kleinfelchen }\end{array}$ & Grossfelchen & $\begin{array}{l}\text { Vierwaldstättersee } \\
\text { Kleinfelchen }\end{array}$ & Grossfelchen \\
\hline Laichfische & $1: 1$ & $3,1: 1$ & $1,2: 1$ & $1: 1$ \\
Alle Fische & $1: 1$ & $2,8: 1$ & $0,9: 1$ & $2: 1$ \\
\hline
\end{tabular}

Die zahlenmässige Dominanz der Männchen bei den Grossfelchen hat insofern eine negative Auswirkung auf künstliche Besatzmassnahmen, als die Eiermenge einer der ausschlaggebenden Faktoren bei der Besatzfischzucht ist.

\subsection{Eintritt der Geschlechtsreife}

Ende 1977 und Anfang 1978 wurden im Alpnachersee total 55 Kleinfelchen in Netzen von 20 und $24 \mathrm{~mm}$ Maschenweite gefangen. Diese Fische, sowohl Männchen als auch Weibchen, waren alle laichreif und zwei Jahre alt. Sie hatten eine mittlere Länge von $21 \mathrm{~cm}$ und ein mittleres Gewicht von 63,1 g. Entsprechend wurden auch bei den älteren Kleinfelchen in der Laichzeit nie juvenile Individuen mit unentwickelten Gonaden gefunden.

Die Grossfelchen des Alpnachersees werden ebenfalls zum grössten Teil im zweiten Lebensjahr geschlechtsreif. Sie haben zu dieser Zeit eine mittlere Länge von $28,3 \mathrm{~cm}$ und ein mittleres Gewicht von 202 g. Von den 201 untersuchten Grossfelchen waren nur 10 Fische juvenil und hätten wahrscheinlich noch nicht während des Fangjahres, sondern erst im dritten Jahr gelaicht. Der Eintritt der Geschlechtsreife erfolgt bei beiden Geschlechtern im selben Alter.

Im Vergleich zu früheren Angaben werden also die heutigen Kleinfelchen des Alpnachersees zwei Jahre früher geschlechtsreif (Birrer und Schweizer [4]). Nach Steinmann [18] waren die «winterlaichenden Grossfelchen» des Alpnachersees im Alter von 2 bis 3 Jahren noch unreif. Im Vierwaldstättersee wurden zu wenig junge 
Fische gefangen, damit man auch hier verlässliche Angaben über den Eintritt der Geschlechtsreife hätte machen können.

\section{Konsequenzen für die Bewirtschaftung der Felchen im Alpnachersee}

Die minimale Netzmaschenweite für den Fischfang im ganzen Vierwaldstättersee beträgt $28 \mathrm{~mm}$ (sogenannte Albelinetze). Als eigentliche Grossfelchennetze verwendet man Grund- und Schwebnetze von 40 bis $50 \mathrm{~mm}$ Maschenweite. Das Fangmindestmass für die Kleinfelchen des gesamten Vierwaldstättersees beträgt heute $25 \mathrm{~cm}$, für die Grossfelchen $30 \mathrm{~cm}$.

\subsection{Der Einfluss unterschiedlicher Maschenweiten}

Um die optimalen Maschenweiten für die zukünftige Bewirtschaftung der Felchenbestände im Alpnachersee festlegen zu können, wurden Versuchsfänge mit Grundund Schwebnetzen verschiedener Maschenweiten durchgeführt. Die Längenvertei-
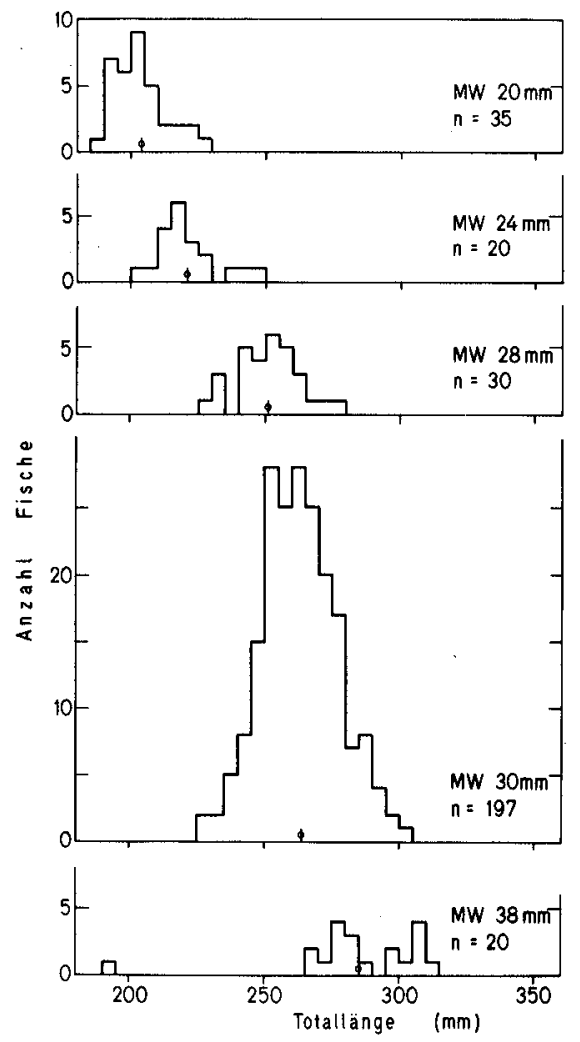

Abb. 8. Längenverteilung der Kleinfelchen des Alpnachersees in Netzen unterschiedlicher Maschenweite. $\phi=$ mittlere Länge (mean length).

Figure 8. Length frequency distribution of 'Kleinfelchen' of Lake Alpnach according to mesh size. 
lungen dieser Fische sind in Abb. 8 und 9 wiedergegeben, was die Selektivität der unterschiedlichen Maschenweiten illustriert. Die mittlere Länge der untersuchten Kleinfelchen aus Netzen mit Maschenweiten von $28 \mathrm{~mm}$ und mehr beträgt $26,4 \mathrm{~cm}$, ihr mittleres Gewicht $155 \mathrm{~g}$. Leider fehlten die Grossfelchen, die zweifelsfrei als solche identifiziert werden konnten, in den 28-mm-Netzen. Beim Jahrgang 1977, dessen Trennung in Klein- und Grossfelchen Schwierigkeiten bereitete (s.oben), stammten 26,3\% der gefangenen Fische aus Netzen mit $28 \mathrm{~mm}$ Maschenweite. Diese im Sommer gefangenen Felchen waren alle $1+$ und hätten zum grössten Teil im gleichen Jahr gelaicht. Ob diese Fische mehrheitlich der gross- oder der kleinwüchsigen Form angehörten, muss aber, wie erwähnt, offengelassen werden.

Es ist aufgrund unserer Erhebungen offensichtlich, dass die Grossfelchen des Alpnachersees ein Jahr zu früh weggefangen werden, d.h. vor dem erstmaligen Ablaichen. Der weitaus grösste Teil der Nachkommen der Grossfelchen stammt zudem heute von Erstlaichern. Nach Nümann [13] und Koelbing [11] sind jedoch die Laichprodukte solcher Fische von geringerer Qualität als jene von älteren Fischen. Eine mindestens teilweise Schonung der Zweitlaicher ist daher bei den Grossfelchen grundsätzlich anzustreben.

\subsection{Bewirtschaftungsmassnahmen}

Die Grossfelchen des Alpnachersees sind seit etwa drei Jahrzehnten die wirtschaftlich wichtigsten Fische dieses Sees. Es liegt deshalb auf der Hand, dass die fischereiliche Bewirtschaftung des Alpnachersees in der Zukunft in erster Linie auf die Grossfel-

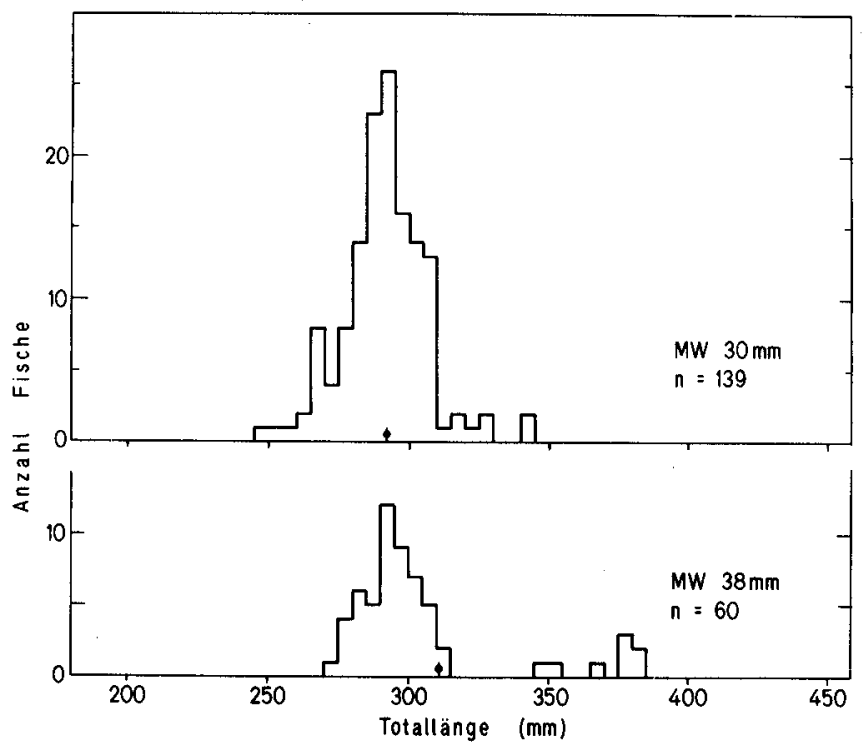

Abb. 9. Längenverteilung der Grossfelchen des Alpnachersees in Netzen unterschiedlicher Maschenweite. $\phi=$ mittlere Länge (mean length).

Figure 9. Length frequency distribution of 'Grossfelchen' of Lake Alpnach according to mesh size. 
chen ausgerichtet werden sollte. Folgende Massnahmen müssten hierfür ins Auge gefasst werden:

- Verbot der sogenannten Albelischwebnetze von $28 \mathrm{~mm}$ Maschenweite. Eine bedeutende Menge junger, nicht geschlechtsreifer Felchen, höchstwahrscheinlich Grossfelchen, wird in diesen Netzen gefangen.

- Einschränkung der Verwendung sogenannter Albeligrundnetze $(28-30 \mathrm{~mm}$ Maschenweite) während der Schonzeit. Der grösste Teil des Grossfelchenmaterials stammt aus solchen Netzen.

- Erhöhung der Maschenweite für die sogenannten Felchen-Schweb- und Felchen-Grundnetze auf 40-44 $\mathrm{mm}$, bei gleichzeitiger Anhebung des Schonmasses für die Grossfelchen auf mindestens $32 \mathrm{~cm}$.

- Intensivierte Besatzwirtschaft mit Grossfelchen.

Es ist leicht einzusehen, dass diese Vorschläge einer optimalen Nutzung des Albelibestandes im Alpnachersee zuwiderlaufen. Dass aber gerade durch solche Massnahmen - namentlich massive Einsätze - die Grossfelchen in einem eutrophen See erfolgreich gefördert werden können, illustriert das bekannte Beispiel des Sempachersees.

\section{Zusammenfassung}

Im Alpnachersee leben langsam- und raschwüchsige Felchenrassen miteinander vergesellschaftet, wodurch Probleme für die zweckmässige Nutzung der Fischbestände entstanden sind: Raschwüchsige juvenile Felchen werden in Netzen für langsamwüchsige adulte Felchen gefangen. Morphologische und biometrische Analysen (Zahl der Kiemenreusendornen und Seitenlinienschuppen, Flossendimensionen, Esterasezymogramme u.a.) zeitigten wohl gewisse formentypische Unterschiede, ermöglichten aber keine eindeutige Zuweisung der einzelnen Fische zu den beiden Formen. Aufgrund des Wachstums im ersten Lebensjahr hingegen liessen sich die einzelnen Felchen den beiden Typen zuordnen:

- langsamwüchsig, hier «Kleinfelchen» genannt, und

- schnellwüchsig, hier «Grossfelchen» genannt.

Die Bezeichnung «Kleinfelchen» entspricht dem Albeli, während "Grossfelchen» dem Balchen, dem Blaufelchen und anderen eingesetzten schnellwüchsigen Felchen entspricht.

Infolge der Eutrophierung hat sich die Wachstumsrate der Kleinfelchen im Alpnachersee während der letzten 40 Jahre etwa verdoppelt. Im ersten Jahr wachsen sie bedeutend langsamer als die Grossfelchen, nachher ist der Unterschied gering. Wachstum und einzelne morphologische Charakteristika trennen langsam- und schnellwüchsige Populationen im Alpnachersee wie auch im Vierwaldstättersee, nicht aber die entsprechenden Formen aus den beiden Seeteilen.

Die Felchen des Alpnacher- und Vierwaldstättersees laichen von November bis Anfang Januar. Sie werden grösstenteils im zweiten Lebensjahr geschlechtsreif. Die Kleinfelchen werden heute vorwiegend im dritten, die Grossfelchen dagegen schon im zweiten Lebensjahr gefangen. Für die Förderung der wirtschaftlich wichtigeren Grossfelchen im Alpnachersee müssten fischereiwirtschaftliche Massnahmen wie Verbot enger Maschenweiten, Erhöhung des Fangmindestmasses und Intensivierung des Einsatzes junger Grossfelchen eingeleitet werden. 


\section{SUMMARY}

The coregonids of Lake Alpnach

In eutrophic Lake Alpnach, fastgrowing and slowgrowing whitefish (Coregonus spp.) stocks are closely associated. Thus, proper stock utilization is impaired since juvenile fish of the fastgrowing form are caught in gillnets used for catching the adults of the slowgrowing form. Although morphological and biochemical data (number of gillrakers and lateral line scales, fin dimensions, esterase zymograms and others) showed some race specific variation, a definite segregation of the individual fish into the two forms on the basis of these data was not practicable. Based on the growth during the first year of life, however, the individual fish could be alloted to the two growth types:

- slowgrowing, here called 'Kleinfelchen', and

- fastgrowing, here called 'Grossfelchen'.

'Kleinfelchen' corresponds to the local name 'Albeli', while 'Grossfelchen' denominates fastgrowing forms of various origins.

Due to eutrophication, the growth rate of 'Kleinfelchen' has almost doubled during the past 40 years. During their first year of life, they grow considerably slower than 'Grossfelchen'. Afterwards, the difference in growth is small. Growth and some morphological characteristics separate the 'Grossfelchen' population from the 'Kleinfelchen' population in Lake Alpnach as well as in Lake Lucerne, but not the corresponding populations in the two lakes.

The coregonids of Lake Alpnach spawn from November to the beginning of January. They reach sexual maturity at the end of their second year. The 'Kleinfelchen' today are caught mostly during their third year of life, while the 'Grossfelchen' are already caught in their second year. In order to enhance the stocks of the commercially more important 'Grossfelchen' in Lake Alpnach, management measures like abolition of small mesh sizes, raising the legal size of the fish, and intensified stocking with young 'Grossfelchen' will have to be envisaged.

\section{RESUME}

Les corégones du lac d'Alpnach

Dans le lac d'Alpnach vivent deux races de corégones dont le taux de croissance est différent, ce qui pose des problèmes pour un aménagement adéquat des populations. Les corégones juvéniles à forte croissance sont capturés dans des filets destinés à des adultes à faible croissance. Des analyses morphologiques et biométriques (nombre de branchiospines et d'écailles sur la ligne latérale, dimensions des nageoires, le zymogramme d'estérase, etc.) montrent bien certaines différences typiques, mais ne permettent pas une attribution individuelle de chaque poisson à l'une ou l'autre des deux formes. Par contre, sur la base de leur taux de croissance pendant la première année, chaque corégone peut se ranger dans l'un des deux types:

- les corégones à faible croissance, appelés ici «Kleinfelchen»,

- les corégones à forte croissance, appelés ici «Grossfelchen».

La dénomination "Kleinfelchen" correspond au nom local de Albeli (Bondelle), tandis que "Grossfelchen» correspond à Balchen (Palée), Blaufelchen (Lavaret) et autres corégones à croissance forte introduits.

Par l'eutrophisation, le taux de croissance des «Kleinfelchen» a environ doublé pendant ces 40 dernières années. Pendant la première année, ils croissent visiblement plus lentement que les «Grossfelchen», plus tard la différence est moindre. Sur la base de la morphologie et de la croissance, on peut distinguer les deux formes de corégones dans le bassin d'Alpnach aussi bien que dans le bassin de Lucerne, mais les deux formes de corégones d'un bassin ne se distinguent pas des deux formes de l'autre bassin.

Les corégones du lac d'Alpnach et ceux du lac des Quatre-Cantons frayent de novembre à début janvier. Ils atteignent leur maturité sexuelle pendant leur deuxième année. Les «Kleinfelchen» sont en grande partie capturés pendant leur troisième année, tandis que les "Grossfelchen" sont par contre déjà capturés pendant leur deuxième année. Pour favoriser la population économiquement plus importante des "Grossfelchen» du lac d'Alpnach, on devrait prendre des mesures piscicoles comme l'interdiction des petites mailles, l'augmentation de la taille minimale de capture et l'intensification des immersions d'alevins de "Grossfelchen». 


\section{VERDANKUNGEN}

Die Direktion der Eidgenössischen Anstalt für Wasserversorgung, Abwasserreinigung und Gewässerschutz (EAWAG), die Eidgenössische Stipendienkommission sowie die Schweizerische Vereinigung der Freunde Finnlands ermöglichten in verdankenswerter Weise die Durchführung der vorliegenden Arbeit. Besonderer Dank gebührt dem Leiter des Fachbereiches Fischereiwissenschaften der EAWAG, Herrn Dr. W. Geiger. Herr J. Bel leistete bei den Altersbestimmungen wertvolle Hilfe. Den Fischereiverwaltungen der Kantone Luzern, Nidwalden und Obwalden sowie den Berufsfischern des Alpnacher- und Vierwaldstättersees sei für ihre Unterstützung gedankt.

\section{LITERATURVERZEICHNIS}

1 Baccini, P.: Untersuchungen über den Schwermetallhaushalt in Seen. Schweiz. Z. Hydrol. 38, 121158 (1976).

2 Bilton, H.T.: Effects of starvation and feeding on circulus-formation on scales of young sockeye salmon of four racial origins, and of one race of young kokanee, coho and chinook salmon. In: Bagenal, T. B. (Hrsg.): Aging of Fish, 234 S. Gresham Press, Surrey 1973.

3 Birrer, A., und Schweizer, W.: Der Weissfisch des Vierwaldstättersees (Coregonus exiguus albellus, Fatio). Mitt. naturf, Ges. Luzern 12, 1-88 (1935).

4 Birrer, A., und Schweizer, W.: Der Weissfisch (Coregonus exiguus albellus, Fatio) im Alpnachersee. Schweiz. FischZtg. 44 (11/12) (1936).

5 Birrer, A., und Schweizer, W.: Der Edelfisch des Vierwaldstättersees (Coregonus Wartmanni nobilis, Fatio). Arch. Hydrobiol. 29, 671-663 (1936).

6 Birrer, A., und Schweizer, W.: Der Balchen des Vierwaldstättersees (Coregonus Schinzii helveticus var. lucernensis, Fatio). Z. Hydrol. 8, 1-2 (1938).

7 Ferguson, A.: The genetic relationships of the coregonid fishes of Britain and Ireland indicated by electrophoretic analysis of tissue proteins. J. Fish Biol. 6, 311-315 (1974).

8 Hartmann, J., und Knoepfler, G.: Zur Unterscheidung von Blaufelchen und Gangfisch des Bodensees. Fischwirt (AFZ) 27 (9), 49-50 (1977).

9 Karbe, L.: Die Chromosomenverhältnisse bei den Coregonen des Bodensees und einiger weiterer voralpiner Seen, ein Beitrag zum Problem der Spezifikation in der Gattung Coregonus. Z. zool. Syst. Evolutionsforsch. 2, 18-40 (1964).

10 Krummenacher, T.: Die Nährstoffbilanz des Alpnachersees. Diss. ETH Zürich Nr.5689, $135 \mathrm{~S}$. (1976).

11 Koelbing, A.: Der Starnberger See und die seinem Trophiezustand angemessene Bewirtschaftungsweise des Coregonenbestandes. Veröff. zool. StSamml., Münch. 17, 1-108 (1974).

12 Lea, E.: On the methods in the herring investigations. Publ. Circonst. Cons. int. Explor. Mer 53, 7 174 (1910).

13 Nümann, W.: The «Blaufelchen» of Lake Constance (Coregonus wartmanni) under negative and positive influence of man. In: Lindsey, C.C., und Woods, C.S. (Hrsg.): Biology of Coregonid fishes, $560 \mathrm{~S}$. University of Manitoba Press, Winnipeg 1970.

14 Pearson, R.E.: Number of circuli and time of annulus formation on scales of Pink Salmon (Oncorhynchus gorbuscha). J. Fish. Res. Bd Canada 23 (5), 747-756 (1966).

15 Rufli, H.: Die heutigen sympatrischen Felchenpopulationen (Coregonus spp.) des Thuner- und Bielersees und ihre Morphologie. Schweiz. Z. Hydrol 40, 7-31 (1978).

16 Rufli, H.: Ernährung und Wachstum der Felchenpopulationen (Coregonus spp.) des Thuner- und Bielersees. Schweiz. Z. Hydrol. 41, 64-93 (1979).

17 Skog, S.: Elektroforetiska populationsundersökningar av sympatriskt levande vandringssik och havssik fran Bottniska viken. Pro gradu Abo Akademi (1977).

18 Steinmann, P.: Monographie der schweizerischen Koregonen. Schweiz. Z. Hydrol. 12 (1/2), 13 (1), (1950/51).

19 Svaerdson, G.: Significance of introgression in Coregonid evolution. In: Lindsey, C.C., und Woods, C.S. (Hrsg.): Biology of Coregonid fishes, 560 S. University of Manitoba Press, Winnipeg 1970.

20 Tesch, F.W.: Age and Growth. In: Ricker, W.E. (Hrsg.): Methods for Assessment of Fish Production in Fresh Waters, 313 S. Blackwell Scientific Publications, Oxford und Edinburgh 1968.

Adresse der Autoren: Seeforschungslaboratorium der EAWAG/ETH, CH-6047 Kastanienbaum. 